\title{
Mithramycin interacts with core histones and modulates epigenetic modifications
}

\author{
Amrita Banerjee*, Chandrima Das, Dipak Dasgupta \\ From Epigenetics \& Chromatin: Interactions and processes \\ Boston, MA, USA. 11-13 March 2013
}

\section{Background}

Mithramycin (MTR), a clinically approved DNA-binding antitumor antibiotic has been found to cross the bloodbrain barrier and is in its preclinical trials in Huntington's disease (HD) [1]. It improved altered nucleosome homeostasis in HD mice, normalizing the chromatin pattern. It has the ability to rebalance epigenetic histone modifications. The binding properties of MTR with histone proteins have been examined from the perspective of the current proposition from our laboratory to classify small DNA binding molecules in terms of their ability to bind chromosomal DNA alone (single binding mode) or both histones and chromosomal DNA (dual binding mode) $[2,3]$.

\section{Materials and methods}

Chemicals were from Sigma, histones from New England Biolabs, antibodies from Abacm, Active Motif. Spectrofluorimetry, circular dichroism (CD) studies, in vitro histone acetyl transferase (HAT) assay and western blot analysis were employed as experimental tools.

\section{Results}

Spectrofluorimetric measurements and CD studies show that MTR binds with micromolar dissociation constant to core histone assembly and its individual histone components. A single isoelliptic point in the CD spectra indicates formation of one type of complex between MTR and histones. Complex formation leads to decrease in molar ellipticity in the far UV CD spectra of core histones. These results led us to investigate the role of MTR as a potential epigenetic modulator. In vitro HAT assays using CREBbinding protein (CBP) HAT domain, a transcriptional coactivator, showed that MTR inhibits H3 K18 acetylation (specifically mediated mark by $\mathrm{CBP} / \mathrm{p} 300$ in vivo) in $\mathrm{H} 3.3$

Biophysics Division, Saha Institute of Nuclear Physics, Block-AF, Sector-1, Bidhan Nagar, Kolkata - 700064, West Bengal, India recombinant histone whereas it induces $\mathrm{H} 3 \mathrm{~K} 18$ hyperacetylation in $\mathrm{H} 3.1 / \mathrm{H} 4$ tetramer as well as core histones.

\section{Conclusions}

MTR exhibits dual binding mode since it binds to histone proteins present in chromatin besides DNA [4]. It alters CBP induced H3 K18 acetylation. In contrast to MTRDNA interaction, association of MTR with histones does not require obligatory presence of bivalent metal ions. The interaction of MTR with histones hints at a different scenario in MTR-chromatin interaction. Experiments are in progress to address the effect of MTR on other epigenetic marks. The significance of these in vitro experiments would also be examined by performing cell line based experiments. These data would be useful in understanding the mechanism of action of anticancer drugs as well as in designing new-generation therapeutics.

\section{Acknowledgements}

This work was funded by Department of Atomic Energy, Govt. of India.

Published: 8 April 2013

\section{References}

1. Stack EC, Del Signore SJ, Luthi-Carter R, Soh BY, Goldstein DR, Matson S, Goodrich S, Markey AL, Cormier K, Hagerty SW, et al: Modulation of nucleosome dynamics in Huntington's disease. Hum Mol Genet 2007, 16:1164-1175.

2. Ghosh S, Majumder P, Pradhan SK, Dasgupta D: Mechanism of interaction of small transcription inhibitors with DNA in the context of chromatin and telomere. Biochim Biophys Acta 2010, 1799:795-809.

3. Dasgupta $D$, Majumder $P$, Banerjee $A$ : A revisit of the mode of interaction of small transcription inhibitors with genomic DNA. J Biosciences 2012, 37:475-481.

4. Mir MA, Majee S, Das S, Dasgupta D: Association of chromatin with anticancer antibiotics, mithramycin and chromomycin A3. Bioorg Med Chem 2003, 11:2791-2801.

doi:10.1186/1756-8935-6-S1-P106

Cite this article as: Banerjee et al:: Mithramycin interacts with core histones and modulates epigenetic modifications. Epigentics \& Chromatin 2013 6(Suppl 1):P106. 